\title{
The acute effects of caffeinated versus non-caffeinated alcoholic beverage on driving performance and attention/reaction time
}

\author{
Jonathan Howland ${ }^{1,2}$, Damaris J. Rohsenow ${ }^{3}$, J. Todd Arnedt', Caleb A. Bliss', Sarah K. Hunt', \\ Tamara Vehige Calise', Timothy Heeren', Michael Winter', Caroline Littlefield' \& \\ Daniel J. Gottlieb ${ }^{5}$
}

Boston University School of Public Health, Boston, MA, USA,' Department of Emergency Medicine, Boston University School of Medicine, Boston, MA, USA, ${ }^{2}$ Center for Alcohol and Addiction Studies at Brown University, Providence, RI, USA, ${ }^{3}$ Sleep and Chronophysiology Laboratory, University of Michigan Medical School, Ann Arbor, MI, USA ${ }^{4}$ and VA Boston Healthcare System, West Roxbury, MA, USA ${ }^{5}$

\section{ABSTRACT}

Aims Marketing that promotes mixing caffeinated 'energy' drinks with alcoholic beverages (e.g. Red Bull with vodka) targets young drinkers and conveys the expectation that caffeine will offset the sedating effects of alcohol and enhance alertness. Such beliefs could result in unwarranted risk taking (e.g. driving while intoxicated). The aim of this study was to assess the acute effects of caffeinated versus non-caffeinated alcoholic beverages on a simulated driving task and attention/reaction time. Design We conducted a $2 \times 2$ between-groups randomized trial in which participants were randomized to one of four conditions: beer and non-alcoholic beer, with and without caffeine added. Caffeine was added in the same proportion as found in a commercially available caffeinated beer $(69 \mathrm{mg} / 12 \mathrm{oz}$ of beer at $4.8 \%$ alc. by vol). Participants Participants were 127 non-dependent, heavy episodic, young adult drinkers (age 21-30) who were college students or recent graduates. The target breath alcohol level was $0.12 \mathrm{~g} \%$. Measures Driving performance was assessed with a driving simulator; sustained attention/reaction with the Psychomotor Vigilance Task (PVT). Findings Across the driving and attention/reaction time we found main effects for alcohol, with alcohol significantly impairing driving and sustained attention/reaction time, with mainly large statistical effects; however, the addition of caffeine had no main or interaction effects on performance. Conclusion The addition of caffeine to alcohol does not appear to enhance driving or sustained attention/reaction time performance relative to alcohol alone.

Keywords Alcohol, attention, caffeine, driving, energy drinks, reaction time.

Correspondence to: Jonathan Howland, Department of Community Health Sciences, Boston University School of Public Health, 801 Massachusetts Avenue, Crosstown Center-3rd Floor, Boston, MA 02118, USA. E-mail: jhowl@bu.edu

Submitted 30 June 2010; initial review completed 7 September 2010; final version accepted 14 September 2010

\section{INTRODUCTION}

'Energy drinks' refer to beverages containing caffeine and sometimes other stimulating herbal products. One hundred and thirty of these products contain more than the $0.02 \%$ caffeine limit the US Federal Drug Administration (FDA) requires for soft drinks, typically 80-141 mg caffeine per $8 \mathrm{oz}$ (equivalent to a 5-oz cup of coffee) [1]. As a result of non-traditional youth-oriented marketing strategies (e.g. sponsors of extreme sports, providing free drinks, internet, text-messaging, Facebook, etc.) [2], 31\% of minor teens and $34-51 \%$ of 18-24-year-olds report regular consumption of energy drinks [2]. Marketing includes unsubstantiated claims that these drinks will enhance attention, endurance, performance, weight loss and fun, while reducing performance decrements from fatigue or alcohol [3].

Consumption of energy drinks with alcohol has grown exponentially since 2001 [4], including both premixed caffeinated alcoholic beverages and drinks mixed in bars or by oneself. While some caffeinated beer products were withdrawn from the market after protest from a coalition of US States' Attorney Generals, other premixed caffeinated alcoholic beverages remain commercially available and mixing the drinks oneself is common. Because caffeinated alcoholic beverages (CABs) are 
promoted as enhancing fun at parties or at bars [4], mixing alcohol and energy drinks is likely to continue, even if the pre-mixed products are withdrawn. People who use energy drinks consume alcohol more frequently than people who do not use energy drinks [2,5]; a quarter of college students reported mixing alcohol and energy drinks in the past month [2].

$\mathrm{CABs}$ may increase risk-taking behaviors. By offsetting the sedating effects of alcohol, CABs decrease feelings of intoxication [6,7], which may lead drinkers to believe that they are less impaired than they are, and thereby may induce more alcohol consumption. France and Denmark have banned the sale of energy drinks [4] and the governments of other countries (Canada and Sweden) have issued warnings about mixing energy drinks with alcohol. In 2009, the US FDA issued a statement expressing concerns about the lack of safety data on CABs (13 November 2009), because recent survey data [2] showed that $\mathrm{CAB}$ use correlated with risky behavior among college students. Students who consumed CABs, relative to those who consumed alcohol without caffeine, were more likely to: (1) be taken advantage of sexually; (2) take advantage of another sexually; (3) ride with an intoxicated driver; (4) be hurt or injured; and (5) require medical treatment [2]. Among lighter drinkers, those who consumed CABs were more likely to drive after drinking. Among patrons leaving a bar, those who had consumed CABs were three times more likely than those who drank non-caffeinated alcohol to leave the bar with a breath-alcohol content (BrAC) of $\geq 0.08 \mathrm{~g} \%$ and four times as likely to intend to drive [8].

The evidence is equivocal as to whether caffeine, when administered in a blinded experimental design, reduces alcohol-induced impairment. Some studies have found that caffeine acutely reverses alcohol-related performance impairment on tests of reaction time, divided attention and psychomotor speed, but not on error rates [9-12]. Other studies did not find caffeine to reduce alcohol-induced impairment significantly in motor coordination and/or reaction time [6,13-15]. It has been proposed that caffeine antagonizes alcohol's effects on response execution but not on inhibitory control [16]. The one study of caffeine-alcohol interaction effects on driving in a simulator found caffeine to partially counteract alcohol-induced impairment $(0.085 \mathrm{~g} \% \mathrm{BrAC})$ in brake latency, although performance remained more impaired in both alcohol conditions relative to placebo [17].

The present study was designed to provide a controlled evaluation of the acute effects of caffeine on impairment after drinking to intoxication. Alcohol administration targeted $\mathrm{BrAC}$ of $0.12 \mathrm{~g} \%$, the level associated with hangover in most drinkers [18] (residual effects the next day are not reported here). Caffeine was added to alcoholic and non-alcoholic beer in the same proportion as in commercially available caffeinated beer and performance effects were evaluated using a driving simulator and a test of sustained attention/reaction time, both of which have been shown previously to be sensitive to acute alcohol effects [19-21].

\section{METHODS}

\section{Participants}

Participants were volunteer university students and recent graduates from greater Boston, MA who were between 21 and 30 years of age and met the following criteria: (1) no drinking problems [score $<5$ on the Short Michigan Alcohol Screening Test (SMAST)] [22] and no history of treatment or counseling for chronic alcohol problems; (2) $\geq 5$ drinks on a single occasion ( $\geq 4$ if female) at least once in the 30 days prior to screening; (3) no health problems or current medication use contraindicated for alcohol; (4) no sleep disorders; (5) fluent English; (6) recently graduated from, or currently attending, an institution of higher learning; (7) not working night shifts at a job; (8) not a daily smoker; (9) not traveled across two or more time zones in the prior month; (10) negative pregnancy test and not nursing, if female; (11) $\geq 1$ and $\leq 7$ caffeinated beverages per day; and (12) $\leq 230 \mathrm{lb}$, to avoid caffeine and beverage loading. Females were not screened for menstrual cycle [23-25]. Regular tobacco users were excluded to avoid possible confounding due to nicotine withdrawal because, for safety reasons, participants were not allowed to leave the laboratory to smoke.

Participants were paid $\$ 150$ upon completion of the study, or a pro-rated amount if their participation was terminated. The Institutional Review Boards at Boston Medical Center, Brown University, and the University of Michigan approved this study.

\section{Study site}

The study was conducted at the General Clinical Research Center (GCRC) at the Boston Medical Center campus.

\section{Study design}

We used a randomized between-groups $2 \times 2$ factorial placebo-controlled design. One factor was alcohol versus placebo beverage; the other factor was caffeinated versus non-caffeinated beverage. Thus, participants received either: (1) caffeinated beer; (2) non-caffeinated beer; (3) caffeinated non-alcoholic beer; or (4) non-caffeinated non-alcoholic beer. This allowed investigation of main effects for caffeine and alcohol as well as interaction 
effects between the two substances. Acute effects were assessed on simulated driving and sustained attention/ reaction time following a 30-minute alcohol absorption period.

\section{Recruitment and screening}

Participants were recruited using newspaper advertisements and websites (i.e. FaceBook, Craig's List). Volunteers were screened by telephone and then onsite where they provided informed consent, were examined by a physician and given a study orientation. After the orientation, participants practiced on the driving simulator and the sustained attention/reaction time test to eliminate learning effects.

\section{Pre-session requirements and session procedures}

To reduce potential confounding by sleep deprivation and sleep schedule irregularity, participants were instructed to maintain an 8-hour time in bed schedule for the 3 nights prior to the experimental session, confirmed by daily sleep/wake diary and call-in to a time-stamped answering machine. They were prohibited from napping 24 hours prior to beverage administration; required to pass a pregnancy test (females) and a breath alcohol test; and were required to abstain from alcohol, medications not already approved by the study physician, sleep aids and recreational drugs for 24 hours and caffeine for 8 hours, prior to their experimental session.

A week after screening, participants returned for the experimental session at 4:00 p.m. in groups of up to five. Car keys were collected if participants drove to the site to prevent drunken driving if a participant dropped out of the study during or after alcoholic beverage administration. Following a standardized dinner, participants were screened for breath alcohol and pregnancy (if female). Participants who were compliant with the pre-laboratory regimen and who were not pregnant were randomized to experimental condition. Beverage administration followed the procedures below. Thirty minutes following beverage administration, participants performed the driving simulator and the sustained attention/reaction time test, received snacks, and prepared for bed.

\section{Beverage administration procedures}

The beverage administration procedures followed the guidelines for balanced placebo studies [26] and the Recommended Council Guidelines on Ethyl Alcohol Administration in Human Experimentation [27]. To maintain double-blinding, the research staff who prepared beverages and conducted breath tests were different from those who collected all other measures.
Alcoholic beverage administration targeted 0.12 g\% BrAC, adjusting for sex $(1.068 \mathrm{~g} / \mathrm{kg}$ body weight for men and $0.915 \mathrm{~g} / \mathrm{kg}$ for women), as per Friel [28]. If participants randomized to alcohol did not reach $0.12 \mathrm{~g} \% \mathrm{BrAC}$ after receiving the prescribed amount of beverage, the ratio of obtained versus target BrAC was used to determine the additional amount of beer to be administered. To maintain blinding, the same number of placebo participants was given a matching extra dose. Hurricane High Gravity ${ }^{\mathrm{TM}}$ (8.1\%) beer (Anheuser Busch, St Louis, MO, USA) and Clausthaler ${ }^{\mathrm{TM}}$ nonalcoholic beer $(<0.01 \%)$ (Radeberger Gruppe KG, Frankfurt am Main, Germany) were the beverages. High alcohol-content beer reduces the volume required to attain the targeted dose. Beer controlled with nonalcoholic beer is an effective beverage combination for the balanced placebo design [29]. Participants who received alcoholic beer received a mean of $44 \mathrm{oz}$ of beverage (37 if female), the equivalent of 6.212 -oz. (4.8\% alc. by vol.) cans. Although beer is absorbed more slowly than spirits it was chosen for ecological validity, as most young adults consume beer.

To caffeinate the alcoholic and placebo beverages, $117 \mathrm{mg}$ of tasteless, anhydrous caffeine powder in solution was added to each 12-oz unit via a pre-packaged syringe, prepared by a compounding pharmacy (Pawtuxet Valley Prescription \& Surgical Center, Inc., Coventry, RI, USA). This dose approximates the caffeine content found in a commercially available caffeinated beer $\left(\right.$ Moonshot $^{\circledR}$ ) that contains $4.8 \%$ alcohol by volume and $69 \mathrm{ml}$ of caffeine in each 12-oz bottle. On average, participants who received caffeine received $383 \mathrm{mg}$ of caffeine (338 if female). Our weight exclusion ensured that no participant received more than $650 \mathrm{mg}$ of caffeine.

Beverage administration began at 7:30 p.m., 3 hours after participants completed their meals, and continued until approximately 8:30 p.m. Participants were told that there was a $25 \%$ chance of being assigned to one of the four experimental conditions and that they should not sniff or taste each other's drinks, nor discuss which beverage they believed they had received. As a manipulation check, a blinded staff member was present throughout beverage administration to monitor and record participants' consumption. Participants were breath-tested, using Intoximeters' AlcoSensor-4 (Intoximeters, Inc., St Louis, MO, USA): after they consumed half their prescribed dose; when they consumed their full prescribed dose; after re-dosing (if participants were not at $0.12 \mathrm{~g} \%$ BrAC after finishing their prescribed dose); and every 15 minutes thereafter, until a final breath test was taken. Participants had an 8-hour (11:00 p.m.-7:00 a.m.) sleep opportunity, during which they were monitored for safety by an emergency medical technician (EMT) on staff to the study. 


\section{Measures}

Individual difference measures

Participant demographics were recorded at enrollment. Average daily volume (ADV) of alcohol intake was calculated to establish recent drinking practices using a twoitem alcohol use questionnaire: (1) 'Considering all your drinking times in the past 30 days, about how often did you have any beer, wine or liquor?', Likert-rated from 1 'once a day' to 7 'did not drink' with each point anchored; and (2) 'In the past 30 days, on a typical day that you drank, about how much did you have to drink in one day?', rated from 1 to 8 , with choices of ' 1 to 7 drinks' and ' 8 or more drinks'. (One drink was defined as $12 \mathrm{oz}$ of beer or wine cooler, $4 \mathrm{oz}$ of wine or $1 \mathrm{oz}$ of liquor.) $\mathrm{ADV}$ is the product of the two.

\section{Simulated driving task}

The 30-minute driving task, sensitive to sleepiness and alcohol [21,30,31], was run on a computer with DriveSim 3.00 software (DriveSim; York Computer Technologies, Kingston, Ontario, Canada), peripheral steering wheel, accelerator and brake. The task presented a driver's orientation of a two-lane highway with lane markings, speed signs, small trees along the roadside and other vehicles. Instructions were to stay in the right lane center and follow a fixed speed limit (60 mph) while 'driving', except when road signs indicated different speed zones. At random intervals, 'wind' pushed the simulated vehicle to the right or left. Off-road excursions elicited a beep and the car was automatically placed back onto the road. The primary dependent variables were participant's: mean speed deviation (current speed minus posted speed in mph); standard deviation of speed deviation (speed variability); mean lane position deviation (center of right lane minus car's current road position in feet); standard deviation of lane position deviation (lane variability); and sum of collisions and roadway departures (crashes).

\section{Sustained attention/reaction time}

This critical component of safe driving was measured using the Psychomotor Vigilance Task (PVT; Ambulatory Monitoring, Inc, Ardsley, New York, USA). The PVT is a hand-held 10-minute visually sustained attention test shown to be sensitive to alcohol effects $[19,20]$. Participants pressed a button as quickly as possible in response to numbers scrolling on the LCD screen, with a random 2-10-second inter-stimulus interval. Response time was counted in milliseconds by a solid-state storage unit for downloading to a computer. The primary outcome variable was median reaction time [32].
Self-reported estimate of blood alcohol concentration (BAC)

To determine whether the coadministration of alcohol and caffeine affects perceptions of intoxication, relative to alcohol alone, participants estimated their blood alcohol level following beverage administration and absorption period on a scale from 0 to $0.15 \mathrm{~g} \%$ using a questionnaire we developed for previous studies, the Self Estimate of Blood Alcohol Concentration (SEBAC).

\section{Data analysis approach}

All measures were examined for normality and outliers. Speed and lane variability were log-transformed to correct for non-normality in the data. Outliers were recoded following recommendations by Tabachnick \& Fidell [33]. Analysis of variance $(2 \times 2)$ investigated the main effects of alcohol and caffeine and their interaction on the speed and lane variables of the simulated driving task and on median PVT reaction time. Effect sizes are reported in terms of Cohen's $f$ [34]), where $f<0.25$ is small, between 0.25 and 0.39 is medium, and 0.40 and above is large. A negative binomial regression model for count data, with a log offset to account for minor variations in length of simulator run time, was used to analyze crashes on the driving simulator.

With a targeted sample of 133 participants available for analysis, our study had $80 \%$ power of detecting the anticipated medium-sized main effect of acute alcohol or caffeine on simulated driving performance.

\section{RESULTS}

\section{Participant enrollment and characteristics}

Two hundred and twenty-four volunteers were screened by telephone, of whom 49 (21.8\%) did not meet inclusion criteria and another 21 (9.4\%) were eligible but subsequently declined participation. Of 154 who signed consent, $12(7.8 \%)$ did not present for the experimental sessions and 13 were excluded because of an error in caffeine administration. Of the 129 participants who completed the study, eight were eliminated from the analysis of the driving simulator data because of technical problems with the simulator and two were excluded because they had difficulty operating the PVT. Thus, 121 participants were available for the analysis of simulated driving performance and 127 participants were available for PVT analysis.

Fifty-three per cent of participants were male; $75 \%$ were white; $10 \%$ were Hispanic; $83 \%$ were students and $17 \%$ were recent graduates. Participants' mean age was 22.9 [standard deviation $(\mathrm{SD})=2.23$; range $21-30$ years]; $\mathrm{ADV}$ was 1.60 ( $\mathrm{SD}=1.03$; range $0.10-4.74$ drinks); mean days buzzed in last 30 days was 7.2 
$(\mathrm{SD}=5.04$; range 1.5-25.8 days). Mean peak BrAC for participants receiving alcohol was $0.12 \mathrm{~g} \%(\mathrm{SD}=$ $0.012)$; range $0.08-0.16 \mathrm{~g} \%$ ). There were no significant differences across the four experimental groups on these variables.

\section{Dependent measures of objective effects}

\section{Speed deviation}

The effect of alcohol was borderline significant, $F_{(1,117)}=2.78, P=0.10$, with a small effect size $f=0.15$ (see Table 1 for means). Mean speed deviation was 3.8 $(\mathrm{SD}=7.8) \mathrm{mph}$ after alcohol compared to $1.6(\mathrm{SD}=5.1)$ mph after non-alcoholic beer. No significant effect of caffeine $\left(F_{(1,117)}=0.01\right.$, effect size $\left.f=0.01\right)$ and no significant interaction $\left(F_{(1,117)}=1.58\right.$, effect size $\left.f=0.11\right)$ was found.

\section{Speed variability}

The effect of alcohol was significant, $F_{(1,117)}=11.92$, $P<0.001$, with a medium effect size $f=0.32$ (see Table 1 for means). Speed variability was $10.8(\mathrm{SD}=7.9) \mathrm{mph}$ after alcohol compared to $6.7(\mathrm{SD}=3.3) \mathrm{mph}$ after non-alcoholic beer. No significant effect of caffeine $\left(F_{(1,117)}=0.22\right.$, effect size $\left.f=0.04\right)$ and no significant interaction ( $F=0.15$, effect size $f=0.03$ ) were found.

\section{Lane position deviation}

The effect of alcohol was not significant, $F_{(1,117)}=0.48$, with a very small effect size $f=0.06$ (see Table 1 for means). No significant effect of caffeine $\left(F_{(1,117)}=0.13\right.$, effect size $f=0.03$ ) and no significant interaction $\left(F_{(1,117)}=0.20\right.$, effect size $\left.f=0.04\right)$ were found.

\section{Lane position variability}

The effect of alcohol was significant, $F_{(1,117)}=22.09$, $P<0.001$, with a large effect size $f=0.43$ (see Table 1 for means). Mean standard deviation for lane deviation was $1.43(\mathrm{SD}=0.55)$ feet after alcohol compared to $1.09(\mathrm{SD}=0.41)$ feet after non-alcoholic beer. The effect of caffeine was borderline significant $\left(F_{(1,117)}=2.89\right.$, $P=0.09$, with a small effect size $f=0.14$ ). Mean standard deviation for lane deviation was $1.21(\mathrm{SD}=0.54)$ feet after caffeine compared to $1.31(\mathrm{SD}=0.50)$ feet after non-caffeinated beverage. No significant interaction $\left(F_{(1,117)}=2.44\right.$, effect size $\left.f=0.13\right)$ was found.

\section{Crashes}

No significant interaction $\left[\chi^{2}(\mathrm{df}=1)=2.34\right]$ was found [see Table 1 for means and relative risk (RR) values]. The effect of alcohol in the main effects model was significant $\left[\chi^{2}(\mathrm{df}=1)=14.79\right]$, indicating an increased risk of crashes after alcohol compared to after non-alcoholic beer. The effect of caffeine in the main effects model was not significant $\left[\chi^{2}(\mathrm{df}=1)=2.60\right]$. Comparing alcohol with caffeine to alcohol without caffeine, the relative risk of crashes was not increased significantly [RR $=0.81$; 95\% confidence interval (CI): 0.28-2.28].

\section{Sustained attention/reaction time}

The effect of alcohol was significant, $F_{(1,123)}=52.30$, $P<0.001$, with a large effect size $f=0.64$ (see Table 1 for

Table 1 Acute effects of alcohol and caffeine beverage manipulations on speed (mph) and lane (feet) deviation means and average standard deviations (SD) and on attention/reaction time (milliseconds).

\begin{tabular}{|c|c|c|c|c|}
\hline & \multicolumn{2}{|l|}{ Alcoholic beer } & \multicolumn{2}{|l|}{ Non-alcoholic beer } \\
\hline & Caffeine & No caffeine & Caffeine & No caffeine \\
\hline & Mean (SD) or RR and CI & Mean (SD) or RR and CI & Mean (SD) or RR and CI & Mean (SD) \\
\hline & $n=28$ & $n=35$ & $n=26$ & $n=32$ \\
\hline Speed deviation mean ${ }^{\mathrm{a}}$ & $2.9(6.5)$ & $4.5(8.7)$ & $2.4(5.1)$ & $1.0(4.9)$ \\
\hline Speed deviation $\mathrm{SD}^{\mathrm{b} . \mathrm{e}}$ & $10.1(7.0)$ & $11.3(8.7)$ & $6.7(3.4)$ & $6.6(3.3)$ \\
\hline Lane deviation mean & $-1.25(0.72)$ & $-1.36(0.85)$ & $-1.22(0.53)$ & $-1.21(0.77)$ \\
\hline Lane deviation $\mathrm{SD}^{c, e}$ & $1.43(0.61)$ & $1.43(0.51)$ & $0.98(0.31)$ & $1.19(0.46)$ \\
\hline Crashes & $2.07(3.58)$ & $2.57(5.57)$ & $0.15(0.37)$ & $0.78(1.54)$ \\
\hline Relative risk ${ }^{\mathrm{f}}$ & 2.65 & 3.29 & 0.20 & Reference \\
\hline \multirow[t]{2}{*}{$95 \% \mathrm{CI}$} & $0.87-8.05$ & $1.14-9.41$ & $0.05-0.86$ & \\
\hline & $n=29$ & $n=36$ & $n=27$ & $n=35$ \\
\hline PVT median reaction time ${ }^{\mathrm{d}}$ & $278.7(46.6)$ & $293.3(47.0)$ & $233.1(22.3)$ & $241.8(25.6)$ \\
\hline
\end{tabular}

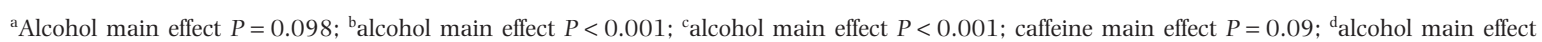
$P<0.001$; caffeine main effect $P=0.09$; ${ }^{\text {data }}$ presented is in non-transformed units; ' relative risk (RR) of crash or off-road event and confidence intervals (CI); PVT: Psychomotor Vigilance Task; RR: relative risk; SD: standard deviation. 
means). Median reaction time was $286.8(\mathrm{SD}=47.0)$ milliseconds after alcohol compared to $238.0(\mathrm{SD}=24.4)$ milliseconds after non-alcoholic beer. The effect of caffeine was borderline significant $\left(F_{(1,123)}=3.02, P=0.09\right.$, with a small effect size $f=0.13$ ). Mean median reaction time was $256.7(\mathrm{SD}=43.2)$ milliseconds after caffeine compared to $267.9 \quad(\mathrm{SD}=45.8)$ milliseconds after non-caffeinated beverage. No significant interaction $\left(F_{(1,117)}=0.20\right.$, effect size $\left.f=0.03\right)$ was found.

\section{Self-reported estimate of $B A C$}

There was no difference in the estimate of $\mathrm{BrAC}$ between participants who received caffeinated beer $(0.11 \pm$ $0.02 \mathrm{~g} \%)$ and non-caffeinated beer $(0.10 \pm 0.02 \mathrm{~g} \%)$ BrAC.

\section{DISCUSSION}

Our findings indicate that under acute intoxication caffeine, in the amounts found typically in CABs, does not reduce alcohol-related impairment in simulated driving or sustained attention/reaction time. Alcohol intoxication resulted in more variability in speed and lateral movements and increased crashes. However, there appears to be little or no protective benefit from the addition of caffeine to alcohol with respect to the safe execution of activities that require sustained attention with rapid accurate decisions. These findings do not appear to result from insufficient statistical power, as the interactions effect sizes were all very small and not of a meaningful size. These findings are consistent with those of Liguori \& Robinson [17], who did not find large effects of caffeinated versus non-caffeinated alcohol on braking latency in a driving simulator or on choice reaction time. Other investigators, however, found that CABs decreased self-perceived intoxication $[6,7]$. We did not find differences in self-estimated BrAC among those who received alcohol with and without caffeine, suggesting that people can evaluate their level of intoxication accurately following consumption of these beverages.

These findings are important within the context of the current interest in the safety of CABs by the US FDA and regulatory agencies in other countries. Despite marketing claims, and consistent with a number of other studies reviewed above, adding caffeine to alcohol has no beneficial effects on performance relevant to safety at these intoxicating doses. Given that expecting beneficial effects from caffeine worsens performance after alcohol, when people are told that they have caffeine added to alcohol [35-37], harmful effects may occur in the natural environment where the beverages are consumed, with the expectation that caffeine will offset alcohol's impairing effects.
The results of this study suggest that public education, via media and warning labels, should be considered regarding the safety of $\mathrm{CABs}$ and that regulators should scrutinize energy drinks and $\mathrm{CAB}$ advertising activities as they relate to promoting safety-related expectancies.

The limitations of this study included a restricted age range, using only one dose of alcohol and of caffeine, and not assessing decision to drive. Results may be different at lower alcohol, or higher caffeine, levels. Further research should examine whether, relative to non-caffeinated alcoholic beverages, CABs increase urge to drink more and affect risk-taking decision making.

\section{Clinical trial registration}

ClinicalTrials.Gov Identifier: NCT00515294.

\section{Declarations of interest}

None.

\section{Acknowledgements}

We wish to acknowledge Sigmund Gurnick whose energy, enthusiasm and sense of humor contributed to the completion of this study. 'Ziggy' died of cancer at age 23 before the preparation of this report. He is greatly missed by his friends and colleagues. This research was supported by a grant from the Centers for Disease Control and Prevention (CDC), grant no. 1R49CE000946 and by the National Center for Research Resources (NCRR), grant no. M01 RR00533, a component of the National Institutes of Health $(\mathrm{NIH})$. Its contents are solely the responsibility of the authors and do not necessarily represent the official view of the NCRR or CDC.

\section{References}

1. Pronsky Z. M. Food Medication Interaction, 10th edn. Pottstown: J.P. Crowe; 1997.

2. O'Brien M. C., McCoy T.P., Rhodes S. D., Wagoner A., Wolfson M. Caffeinated cocktails: energy drink consumption, highrisk drinking, and alcohol-related consequences among college students. Acad Emerg Med 2008; 15: 453-60.

3. Reissig C. J., Strain E. C., Griffiths R. R. Caffeinated energy drinks—a growing problem. Drug Alcohol Depend 2009; 99: $1-10$.

4. Simon M., Mosher J. Alcohol, Energy Drinks, and Youth: A Dangerous Mix. San Rafael, CA: Marin Institute; 2007.

5. Arria A. M., Caldeira K. M., Kasperski S. J., O'Grady K. E., Vincent K. B., Griffiths R. R. Increased alcohol consumption, nonmedical prescription drug use and illicit drug use are associated with energy drink consumption among college students. J Addict Med 2010; 4: 74-80.

6. Ferreira S. E., de Mello M. T., Pompeia S., de SouzaFormigoni M. L. Effects of energy drink ingestion on alcohol intoxication. Alcohol Clin Exp Res 2006; 30: 598-605.

7. Marczinski C. A., Fillmore M. T. Clubgoers and their trendy cocktails: implications of mixing caffeine into alcohol on 
information processing and subjective reports of intoxication. Exp Clin Psychopharmacol 2006; 14: 450-8.

8. Thombs D. L., O'Mara R. J., Tsukamoto M., Rossheim M. E., Weiler R. M., Merves M. L. et al. Event-level analyses of energy drink consumption and alcohol intoxication in bar patrons. Addict Behav 2010; 35: 325-30.

9. Burns M., Moskowitz H. Two experiments on alcoholcaffeine interaction. Alcohol Drugs Driving 1990; 5: 303-15.

10. Fillmore M., Vogel-Sprott M. Behavioral effects of combining alcohol and caffeine: the contribution of drug-related expectancies. Exp Clin Psychopharmacol 1995; 3: 33-8.

11. Franks H. M., Hagedorn H., Hensley V. R., Hensley W. J., Starmer G. A. The effect of caffeine on human performance, alone and in combination with ethanol. Psychopharmacologia 1975 ; 45: 177-81.

12. Hasenfratz M., Bunge A., Dal Pra G., Battig K. Antagonistic effects of caffeine and alcohol on mental performance parameters. Pharmacol Biochem Behav 1993; 46: 463-5.

13. Fillmore M., Vogel-Sprott M. Psychomotor performance under alcohol and under caffeine: expectancy and pharmacological effects. Exp Clin Psychopharmacol 1994; 2: 31928.

14. Fillmore M. T., Roach E. L., Rice J. T. Does caffeine counteract alcohol-induced impairment? The ironic effects of expectancy. J Stud Alcohol 2002; 63: 745-54.

15. Liguori A., Robinson J. H. Caffeine antagonism of alcoholinduced driving impairment. Drug Alcohol Depend 1991; 63: 123-9.

16. Marczinski C. A., Fillmore M. T. Dissociative antagonistic effects of caffeine on alcohol-induced impairment of behavioral control. Exp Clin Psychopharmacol 2003; 11: 228-36.

17. Liguori A., Robinson J. H. Caffeine antagonism of alcoholinduced driving impairment. Drug Alcohol Depend 2001; 63: 123-9.

18. Rohsenow D. J., Howland J., Minsky S. J., Greece J., Almeida A., Roehrs T. A. The Acute Hangover Scale: a new measure of immediate hangover symptoms. Addict Behav 2007; 32: 1314-20.

19. Howland J., Rohsenow D., Greece J., Littlefield C. A., Almeida A. B., Heeren T. et al. The effects of binge drinking on college students' next-day academic test-taking performance and mood state. Addiction 2010; 105: 655-65.

20. Rohsenow D. J., Howland J., Arnedt J. T., Almeida A. B., Greece J., Minsky S. et al. Intoxication with bourbon versus vodka: effects on hangover, sleep, and next-day neurocognitive performance in young adults. Alcohol Clin Exp Res 2010; 34: 1-10.

21. Arnedt J. T., Wilde G. J., Munt P. W., MacLean A. W. How do prolonged wakefulness and alcohol compare in the decrements they produce on a simulated driving task? Accid Anal Prev 2001; 33: 337-44.

22. Selzer M. L., Vinokur A., van Rooijen L. A self-administered Short Michigan Alcoholism Screening Test (SMAST). J Stud Alcohol 1975; 36: 117-26.
23. Brick J., Nathan P. E., Westrick E., Frankenstein W., Shapiro A. The effect of menstrual cycle on blood alcohol levels and behavior. J Stud Alcohol 1986; 47: 472-7.

24. Niaura R. S., Nathan P. E., Frankenstein W., Shapiro A. P., Brick J. Gender differences in acute psychomotor, cognitive, and pharmacokinetic response to alcohol. Addict Behav 1987; 12: 345-56.

25. Terner J. M., de Wit H. Menstrual cycle phase and responses to drugs of abuse in humans. Drug Alcohol Depend 2006; 84: $1-13$.

26. Rohsenow D. J., Marlatt G. A. The balanced placebo design: methodological considerations. Addict Behav 1981; 6: 10722.

27. National Institute on Alcohol Abuse and Alcoholism. National Advisory Council on Alcohol Abuse and AlcoholismRecommneded Council Guidelines on Ethyl Alcohol Administration in Human Experimentation. Revised May 2005. Available at: http://www.niaaa.nih.gov/resources/ researchresources/job22.htm (accessed 21 June 2010; archived by Webcite at http://www.webcitation.org/ 5uWiCAKWP).

28. Friel P. N., Logan B. K., O'Malley D., Baer J. S. Development of dosing guidelines for reaching selected target breath alcohol concentrations. J Stud Alcohol 1999; 60: 555-65.

29. Keane T. M., Lisman S. A., Kreutzer J. Alcoholic beverages and their placebos: an empirical evaluation of expectancies. Addict Behav 1980; 5: 313-28.

30. Arnedt J. T., Owens J., Crouch M., Stahl J., Carskadon M. A. Neurobehavioral performance of residents after heavy night call vs after alcohol ingestion. JAMA 2005; 294: 1025-33.

31. Arnedt J. T., Wilde G. J., Munt P. W., MacLean A. W. Simulated driving performance following prolonged wakefulness and alcohol consumption: separate and combined contributions to impairment. J Sleep Res 2000; 9: 233-41.

32. Van Dongen H. P., Maislin G., Mullington J. M., Dinges D. F. The cumulative cost of additional wakefulness: doseresponse effects on neurobehavioral functions and sleep physiology from chronic sleep restriction and total sleep deprivation. Sleep 2003; 26: 117-26.

33. Tabachnick B. G., Fidell L. S., editors. Using Multivariate Statistics, 3rd edn. New York: Harper and Collins; 1996.

34. Cohen J. Statistical Power Analysis for the Behavioral Sciences. 2nd ed. Hillside, NJ: Erlbaum Associates.

35. Fillmore M. T., Blackburn J. Compensating for alcoholinduced impairment: alcohol expectancies and behavioral disinhibition. J Stud Alcohol 2002; 63: 237-46.

36. Fillmore M. T., Mulvihill L. E., Vogel-Sprott M. The expected drug and its expected effect interact to determine placebo responses to alcohol and caffeine. Psychopharmacology (Berl) 1994; 115: 383-8.

37. Fillmore M. T., Vogel-Sprott M. Evidence that expectancies mediate behavioral impairment under alcohol. J Stud Alcohol 1996; 57: 598-603. 